\title{
Localized boundary-domain integral equation formulation for mixed type problems
}

\author{
Otar Chkadua, Sergey E. Mikhailov and David Natroshvili
}

\begin{abstract}
Some modified direct localized boundary-domain integral equations (LBDIEs) systems associated with the mixed boundary value problem (BVP) for a scalar "Laplace" PDE with variable coefficient are formulated and analyzed. The main results established in the paper are the LBDIEs equivalence to the original variable-coefficient BVPs and the invertibility of the corresponding localized boundary-domain integral operators in appropriately chosen function spaces.
\end{abstract}

Keywords. Partial differential equation, variable coefficients, mixed problem, localized parametrix, localized boundary-domain integral equations, pseudo-differential operators.

2010 Mathematics Subject Classification. 35J25, 31B10, 45K05, 45A05.

\section{Introduction}

Partial Differential Equations (PDEs) with variable coefficients arise naturally in mathematical modelling of non-homogeneous media (e.g. functionally graded materials or materials with damage induced inhomogeneity) in solid mechanics, electro-magnetics, thermo-conductivity, fluid flows trough porous media, and other areas of physics and engineering.

The boundary integral equation method/boundary element method (BIEM/ BEM) is a well established tool for solution of boundary value problems (BVPs) with constant coefficients. The main ingredient for reducing a BVP for a PDE to a BIE is a fundamental solution to the original PDE. However, it is generally not available in an analytical and/or cheaply calculated form for PDEs with variable coefficients. Following Levi and Hilbert, one can use in this case a parametrix (Levi function) as a substitute for the fundamental solution. Parametrix is usually available much easier than a fundamental solution and describes correctly the main part of the fundamental solution although does not have to satisfy the original PDE. This reduces the problem not to a boundary integral equation but to a boundarydomain integral equations (BDIEs) system, see, e.g., $[10,11]$. The discretization

This work was supported by the International Joint Project Grant - 2005/R4 "Boundary-Domain Integral Equations: Formulation, Analysis, Localization" of the Royal Society, UK. 
of the BDIE leads then to a system of algebraic equations of similar size as in the FEM, however the matrix of the system is not sparse as in the FEM, but dense and thus less efficient for numerical solution.

The localized boundary-domain integral equation method (LBDIEM) emerged recently $[8,12,13,15,16]$ addressing this issue and making the BDIE competitive with the FEM for such problems. The LBDIEM employs specially constructed localized parametrices to reduce BVPs with variable coefficients to localized boundary-domain integral or integro-differential equations. After a locallysupported mesh-based or mesh-less discretization this ends up in sparse systems of algebraic equations. Further advancing the LBDIEM requires a deeper analytical insight into the properties of the corresponding integral operators such as the solvability of LBDIE, uniqueness of a solution, equivalence to the original BVPs and the invertibility of LBDIEs. The analysis of non-localized segregated BDIEs is presented in [1,2,5] and that of united BDIDEs in [9].

The analysis of the LBDIE corresponding to the Dirichlet and Neumann BVPs, based on a parametrix localized by multiplying with a cut-off function, were presented in [3] and [4].

In this paper we develop analysis of some direct segregated localized boundarydomain integral equations system (LBDIEs) for mixed type BVPs. Our main goal is to prove:

(i) the equivalence of the LBDIEs to the original mixed type BVPs;

(ii) the invertibility of the corresponding localized boundary-domain integral operators in the appropriately chosen function spaces.

\section{Formulation of the boundary value problem}

Let $\Omega^{+}$be a bounded open three-dimensional region of $\mathbb{R}^{3}$ and $\Omega^{-}:=\mathbb{R}^{3} \backslash \overline{\Omega^{+}}$. For simplicity, we assume that the boundary $S:=\partial \Omega^{+}$is a simply connected, closed, infinitely smooth surface, which is divided into two nonintersecting, simply connected sub-manifolds $S_{D}$ and $S_{N}$ with infinitely smooth boundary curve $\gamma:=\partial S_{D}=\partial S_{N} \in C^{\infty}$. Further let $a \in C^{\infty}\left(\mathbb{R}^{3}\right), a(x)>0$ for $x \in \mathbb{R}^{3}$ and $a(x)=$ const $>0$ for sufficiently large $|x|$.

In this paper we consider the localized boundary-domain integral equations associated with the following scalar elliptic differential equation:

$$
L u(x):=L\left(x, \partial_{x}\right) u(x):=\sum_{i=1}^{3} \partial_{x_{i}}\left(a(x) \partial_{x_{i}} u(x)\right)=f(x), \quad x \in \Omega^{+},
$$


where $u$ is an unknown function and $f$ is a given function in $\Omega^{+}$; here $\partial_{j}=$ $\partial_{x_{j}}:=\partial / \partial x_{j}(j=1,2,3), \partial_{x}=\left(\partial_{x_{1}}, \partial_{x_{2}}, \partial_{x_{3}}\right)$.

In what follows $H_{2}^{s}\left(\Omega^{+}\right), H_{2 \text {,loc }}^{s}\left(\Omega^{-}\right), H_{2}^{s}(S)$ denote the Sobolev-Slobodetski (Bessel potential) spaces.

For $S_{1} \subset S$, we will use the subspace $\widetilde{H}_{2}^{s}\left(S_{1}\right)=\left\{g: g \in H_{2}^{S}(S)\right.$, supp $\left.g \subset \overline{S_{1}}\right\}$ of $H_{2}^{s}(S)$, while $H_{2}^{s}\left(S_{1}\right)=\left\{r_{S_{1}} g: g \in H_{2}^{s}(S)\right\}$ denotes the space of restriction on $S_{1}$ of functions from $H_{2}^{s}(S)$, where $r_{S_{1}}$ denotes the restriction operator on $S_{1}$.

From the trace theorem (see, e.g., [7]) for $u \in H_{2}^{1}\left(\Omega^{+}\right)\left(u \in H_{2, \text { loc }}^{1}\left(\Omega^{-}\right)\right)$it follows that $\left.u\right|_{S} ^{ \pm}:=\tau_{S}^{ \pm} u \in H_{2}^{\frac{1}{2}}(S)$, where $\tau_{S}^{ \pm}$is the trace operator on $S$ from $\Omega^{ \pm}$. We will also use the notation $u^{ \pm}$or $[u]^{ \pm}$for the traces $\left.u\right|_{S} ^{ \pm}$, when this will cause no confusion.

For $u \in H_{2}^{2}\left(\Omega^{+}\right)$, we denote by $T^{ \pm}$the corresponding co-normal differentiation operator on $S$

$$
\begin{aligned}
{[T u]^{ \pm} } & =T^{ \pm} u=T^{ \pm}\left(x, n(x), \partial_{x}\right) u(x):=\sum_{i=1}^{3} a(x) n_{i}(x)\left[\partial_{x_{i}} u(x)\right]^{ \pm} \\
& =a(x)\left[\partial_{n(x)} u(x)\right]^{ \pm},
\end{aligned}
$$

where $n(x)$ is the exterior (to $\Omega^{+}$) unit normal vector at a point $x \in S$ and $\partial_{n(x)}$ denotes the normal derivative operator.

For

$$
u \in H_{2}^{1,0}\left(\Omega^{+}, L\right):=\left\{w \in H_{2}^{1}\left(\Omega^{+}\right): L\left(x, \partial_{x}\right) w \in L_{2}\left(\Omega^{+}\right)\right\}
$$

and

$$
u \in H_{2, \mathrm{loc}}^{1,0}\left(\Omega^{-}, L\right):=\left\{w \in H_{2, \mathrm{loc}}^{1}\left(\Omega^{-}\right): L\left(x, \partial_{x}\right) w \in L_{2, \mathrm{loc}}\left(\Omega^{-}\right)\right\}
$$

we can correctly define a generalized co-normal derivative $T^{ \pm} u \in H_{2}^{-\frac{1}{2}}(S)$ with the help of Green's formula,

$$
\begin{array}{r}
\int_{\Omega^{ \pm}}[v L u+E(u, v)] d x= \pm\left\langle T^{ \pm} u, v^{ \pm}\right\rangle_{S}, \\
\forall v \in H_{2}^{1}\left(\Omega^{+}\right) \quad\left[\forall v \in H_{2, \mathrm{comp}}^{1}\left(\overline{\Omega^{-}}\right)\right],
\end{array}
$$

where

$$
E(u, v):=\sum_{i=1}^{3} a(x) \partial_{x_{i}} u(x) \partial_{x_{i}} v(x),
$$

and $\langle\cdot, \cdot\rangle_{S}$ denote the duality brackets between the spaces $H_{2}^{-\frac{1}{2}}(S)$ and $H_{2}^{\frac{1}{2}}(S)$ which extend the usual $L_{2}(S)$ scalar product. 
In the case of a bounded domain $\Omega^{+}$and for $u \in H_{2}^{1,0}\left(\Omega^{+}, L\right)$ we have the following Green's formulas:

$$
\begin{aligned}
\int_{\Omega^{+}}[v L u+E(u, v)] d x & =\left\langle T^{+} u, v^{+}\right\rangle_{S} \quad \forall v \in H_{2}^{1}\left(\Omega^{+}\right), \\
\int_{\Omega^{+}}[v L u-L v u] d x & =\left\langle T^{+} u, v^{+}\right\rangle_{S}-\left\langle T^{+} v, u^{+}\right\rangle_{S} \forall v \in H_{2}^{1,0}\left(\Omega^{+}\right) .
\end{aligned}
$$

We will consider the LBDIEs approaches for the following mixed type boundary value problem: Find a function $u \in H_{2}^{1}\left(\Omega^{+}\right)$satisfying the conditions

$$
\begin{aligned}
L u=f & \text { in } \Omega^{+}, \\
r_{S_{D}} u^{+}=\varphi_{0} & \text { on } S_{D}, \\
r_{S_{N}} T^{+} u=\psi_{0} & \text { on } S_{N},
\end{aligned}
$$

where

$$
\varphi_{0} \in H_{2}^{\frac{1}{2}}\left(S_{D}\right), \quad \psi_{0} \in H_{2}^{-\frac{1}{2}}\left(S_{N}\right), \quad f \in H_{2}^{0}\left(\Omega^{+}\right) .
$$

Equation (2.4) is understood in the distributional sense, condition (2.5) is understood in the trace sense, while equality (2.6) is understood in the functional sense in accordance with (2.1).

We have the following well-known uniqueness and existence result (see, e.g., [7]).

Theorem 2.1. (i) The homogeneous mixed BVP has only a trivial solution.

(ii) Let the inclusions (2.7) be satisfied. Then the corresponding nonhomogeneous mixed BVP is uniquely solvable.

Proof. The uniqueness result immediately follows from Green's formula (2.2) with $v=u$ as a solution of the corresponding homogeneous boundary value problem. The existence result follows directly from the weak variational formulation of the above problems and the Lax-Milgram theorem.

As we have already mentioned in the Introduction, our main goal is to reduce the mixed BVP to the equivalent localized boundary-domain integral equations and prove the invertibility of the corresponding nonstandard integral operators in appropriately chosen function spaces. 


\section{Localized boundary-domain integral equations approach}

\subsection{Basic integral relations}

Denote by $P_{1}(x, y)$ the parametrix (Levi function) of the operator $L\left(x, \partial_{x}\right)$ considered in [1],

$$
P_{1}(x, y)=-\frac{1}{4 \pi a(y)|x-y|}, \quad x, y \in \mathbb{R}^{3}, x \neq y,
$$

with the property

$$
L\left(x, \partial_{x}\right) P_{1}(x, y)=\delta(x-y)+R_{1}(x, y),
$$

where $\delta(\cdot)$ is the Dirac distribution and

$$
R_{1}(x, y)=\sum_{i=1}^{3} \frac{x_{i}-y_{i}}{4 \pi a(y)|x-y|^{3}} \frac{\partial a(x)}{\partial x_{i}}, \quad x, y \in \mathbb{R}^{3}, x \neq y,
$$

possesses a weak singularity of type $\mathcal{O}\left(|x-y|^{-2}\right)$ for small $|x-y|$.

Let $X_{\varepsilon}^{k}$ be the subclass of cut-off functions introduced in $[3,4]$.

Definition 3.1. We say that $\chi \in X_{\varepsilon}^{k}$ if

$$
\begin{aligned}
\chi & \in C^{k}\left(\mathbb{R}^{3}\right), \quad k \geq 0, \\
\chi(x) & \geq 0 \quad \forall x \in \mathbb{R}^{3}, \\
\chi(0) & =1, \quad \chi(x)=0 \quad \text { for }|x| \geq \varepsilon>0,
\end{aligned}
$$

and $\chi(x)=\widetilde{\chi}(|x|)$, where $\widetilde{\chi}$ is a non-increasing function on $[0,+\infty)$.

Further we define a localized parametrix

$$
P(x, y) \equiv P_{\chi}(x, y)=P_{1}(x, y) \chi(x-y), \quad x, y \in \mathbb{R}^{3},
$$

with $\chi \in X_{\varepsilon}^{k}$.

It is clear that

$$
L\left(x, \partial_{x}\right) P(x, y)=\delta(x-y)+R(x, y),
$$


where $\Delta$ is the Laplace operator, $\Delta=\partial_{1}^{2}+\partial_{2}^{2}+\partial_{3}^{2}$ and

$$
\begin{aligned}
& R(x, y) \equiv R_{\chi}(x, y) \\
&=-\frac{1}{4 \pi a(y)}\left\{a(x)\left[\frac{\Delta_{x} \chi(x-y)}{|x-y|}+2 \sum_{j=1}^{3} \frac{\partial \chi(x-y)}{\partial x_{j}} \frac{\partial}{\partial x_{j}} \frac{1}{|x-y|}\right]\right. \\
&\left.+\sum_{j=1}^{3} \frac{\partial a(x)}{\partial x_{j}} \frac{\partial}{\partial x_{j}} \frac{\chi(x-y)}{|x-y|}\right\}, \quad x, y \in \mathbb{R}^{3} .
\end{aligned}
$$

We see that the function $R(x, y)$ possesses a weak singularity $\mathcal{O}\left(|x-y|^{-2}\right)$.

For $v(x):=P(x, y)$ and $u \in H_{2}^{1,0}\left(\Omega^{+}\right)$, we obtain from (2.3) the third Green's identities,

$$
\begin{aligned}
& u+\mathcal{R}_{+} u-V\left(T^{+} u\right)+W\left(u^{+}\right)=\mathcal{P}_{+}(L u) \text { in } \Omega^{+}, \\
& \mathcal{R}_{+} u-V\left(T^{+} u\right)+W\left(u^{+}\right)=\mathcal{P}_{+}(L u) \text { in } \Omega^{-},
\end{aligned}
$$

where

$$
\begin{aligned}
V g(y) & :=-\int_{S} P(x, y) g(x) d S_{x}, \\
W g(y) & :=-\int_{S}\left[T\left(x, n(x), \partial_{x}\right) P(x, y)\right] g(x) d S_{x}, \\
\mathcal{P}_{ \pm} g(y): & =\int_{\Omega^{ \pm}} P(x, y) g(x) d x, \quad \mathcal{R}_{ \pm} g(y):=\int_{\Omega^{ \pm}} R(x, y) g(x) d x .
\end{aligned}
$$

We denote by $\mathcal{V}, \mathcal{W}$ the direct values of the surface potentials, which generate the boundary operators on $S$, i.e.,

$$
\begin{aligned}
\mathcal{V} g(y) & :=-\int_{S} P(x, y) g(x) d S_{x}, \\
\mathcal{W} g(y) & :=-\int_{S}\left[T\left(x, n(x), \partial_{x}\right) P(x, y)\right] g(x) d S_{x} .
\end{aligned}
$$

Moreover, let

$$
\begin{aligned}
\mathcal{P} g(y) & :=\int_{\mathbb{R}^{3}} P(x, y) g(x) d x, \quad \mathcal{R} g(y):=\int_{\mathbb{R}^{3}} R(x, y) g(x) d x, \\
\mathcal{W}^{\prime} g(y) & :=-\int_{S}\left[T\left(y, n(y), \partial_{y}\right) P(x, y)\right] g(x) d S_{x}, \\
\mathscr{L}^{ \pm} g & :=[T W g]_{S}^{ \pm}, \quad \mathcal{R}_{+}^{ \pm} u:=\left[\mathcal{R}_{+} u\right]_{S}^{ \pm}, \quad \mathcal{P}_{+}^{ \pm} u:=\left[\mathcal{P}_{+} u\right]_{S}^{ \pm} .
\end{aligned}
$$

Due to the results obtained in [4] these operators are well defined. In the next subsection we collect some auxiliary material needed in our analysis (for details see $[1,4])$. 


\subsection{Properties of potentials}

Theorem 3.2. The operators

$$
\begin{gathered}
V: H_{2}^{-\frac{1}{2}}(S) \rightarrow H_{2}^{1}\left(\Omega^{ \pm}\right) \quad \text { with } \chi \in X_{\varepsilon}^{1}, \\
W: H_{2}^{\frac{1}{2}}(S) \rightarrow H_{2}^{1}\left(\Omega^{ \pm}\right) \text {with } \chi \in X_{\varepsilon}^{2}, \\
V: H_{2}^{\frac{1}{2}}(S) \rightarrow H_{2}^{2}\left(\Omega^{ \pm}\right) \text {with } \chi \in X_{\varepsilon}^{2},
\end{gathered}
$$

are continuous. Moreover, supp Vg and supp $W h$ are localized in the closed $\varepsilon$ neighbourhood of the surface $S$, and for $g \in H_{2}^{-\frac{1}{2}}(S)$ and $h \in H_{2}^{\frac{1}{2}}(S)$ we have, $L\left(y, \partial_{y}\right) V g \in H_{2}^{0}\left(\Omega^{ \pm}\right)$if $\chi \in X_{\varepsilon}^{2}$ and $L\left(y, \partial_{y}\right) W h \in H_{2}^{0}\left(\Omega^{ \pm}\right)$if $\chi \in X_{\varepsilon}^{3}$.

Denote by $\mathcal{P}_{0}=a \mathcal{P}$ the pseudodifferential operator of order -2 with the symbol $\lambda(\xi)$,

$$
\begin{aligned}
\mathcal{P}_{0} g & =\mathcal{F}^{-1}\{\lambda(\xi) \mathcal{F} g\}, \\
\lambda(\xi) \equiv \lambda_{\chi}(\xi) & =\mathcal{F}_{x \rightarrow \xi}\left[-\frac{1}{4 \pi} \frac{\chi(x)}{|x|}\right]=-\frac{1}{|\xi|^{2}} \widetilde{\chi}(0)+\frac{1}{|\xi|^{2}} \int_{0}^{\infty} \widetilde{\chi}^{\prime}(\rho) \cos (\rho|\xi|) d \rho .
\end{aligned}
$$

where $\mathcal{F}$ and $\mathcal{F}^{-1}$ are the direct and the inverse Fourier transform. For $\chi \in X_{\epsilon}^{1}$ this implies

$$
c_{1}(1+|\xi|)^{-2} \leq|\lambda(\xi)| \leq c_{2}(1+|\xi|)^{-2}, \quad \xi \in \mathbb{R}^{3}
$$

Then we have the representations

$$
\begin{aligned}
& V_{0} g=a V g=-\mathcal{F}^{-1}\left\{\lambda(\xi) \mathcal{F}\left(g \delta_{S}\right)\right\}=-\mathcal{P}_{0}\left(g \delta_{S}\right), \\
& W_{0} h=a W h=-\mathcal{F}^{-1}\left\{\lambda(\xi) \mathcal{F}\left[-\partial_{n}\left(a h \delta_{S}\right)\right]\right\}=-\mathcal{P}_{0}\left[-\partial_{n}\left(a h \delta_{S}\right)\right],
\end{aligned}
$$

where the singular distributions $\partial_{n}\left(a h \delta_{S}\right)$ and $g \delta_{S}$ are defined as follows [14]:

$$
\begin{aligned}
\left\langle\partial_{n}\left(a h \delta_{S}\right), \varphi\right\rangle_{\mathbb{R}^{3}} & :=-\int_{S} a h \partial_{n} \varphi d S, \\
\left\langle g \delta_{S}, \varphi\right\rangle_{\mathbb{R}^{3}} & :=\int_{S} g \varphi d S \quad \forall \varphi \in \mathcal{D}\left(\mathbb{R}^{3}\right) .
\end{aligned}
$$

Further, denote by $\mathcal{M}$ the pseudodifferential operator of order 2 , the inverse to $\mathcal{P}_{0}$,

$$
\mathcal{M} g=\mathscr{F}^{-1}\{m(\xi) \mathcal{F} g\}, \quad m(\xi)=\frac{1}{\lambda(\xi)} .
$$


The operators

$$
\begin{aligned}
& \mathcal{M}: H_{2}^{t}\left(\mathbb{R}^{3}\right) \rightarrow H_{2}^{t-2}\left(\mathbb{R}^{3}\right) \quad \text { for arbitrary } t \in \mathbb{R}, \\
& \diamond\left(\mathbb{R}^{3}\right) \rightarrow \diamond\left(\mathbb{R}^{3}\right), \\
& \varsigma^{\prime}\left(\mathbb{R}^{3}\right) \rightarrow \varsigma^{\prime}\left(\mathbb{R}^{3}\right), \\
& \mathcal{P}_{0}: H_{2}^{t}\left(\mathbb{R}^{3}\right) \rightarrow H_{2}^{t+2}\left(\mathbb{R}^{3}\right) \quad \text { for arbitrary } t \in \mathbb{R} \text {, } \\
& \diamond\left(\mathbb{R}^{3}\right) \rightarrow \diamond\left(\mathbb{R}^{3}\right), \\
& \delta^{\prime}\left(\mathbb{R}^{3}\right) \rightarrow \delta^{\prime}\left(\mathbb{R}^{3}\right)
\end{aligned}
$$

are invertible. Here $\delta\left(\mathbb{R}^{3}\right)$ and $\wp^{\prime}\left(\mathbb{R}^{3}\right)$ are the Schwartz spaces of rapidly decreasing functions and tempered distributions respectively.

Theorem 3.3. Let $g \in H_{2}^{-\frac{1}{2}}(S)$, and $h \in H_{2}^{\frac{1}{2}}(S)$. Then there hold the following jump relations on $S$ :

$$
\begin{aligned}
{[V g]^{ \pm} } & =\mathcal{V} g, & & \chi \in X_{\varepsilon}^{1}, \\
{[W h]^{ \pm} } & =\mp \frac{1}{2} h+\mathcal{W} h, & & \chi \in X_{\varepsilon}^{2}, \\
{[T V g]^{ \pm} } & = \pm \frac{1}{2} g+\mathcal{W}^{\prime} g, & & \chi \in X_{\varepsilon}^{2}, \\
{[T W h]^{+}-[T W h]^{-} } & =\mathscr{L}^{+} h-\mathscr{L}^{-} h=\frac{\partial a}{\partial n} h, & & \chi \in X_{\varepsilon}^{3} .
\end{aligned}
$$

Theorem 3.4. The operators

$$
\begin{array}{cc}
\mathcal{V}: H_{2}^{-\frac{1}{2}}(S) \rightarrow H_{2}^{\frac{1}{2}}(S), & \chi \in X_{\varepsilon}^{1}, \\
\mathcal{W}: H_{2}^{\frac{1}{2}}(S) \rightarrow H_{2}^{\frac{1}{2}}(S), & \chi \in X_{\varepsilon}^{2}, \\
\mathcal{W}^{\prime}: H_{2}^{-\frac{1}{2}}(S) \rightarrow H_{2}^{-\frac{1}{2}}(S), & \chi \in X_{\varepsilon}^{2}, \\
\mathscr{L}^{ \pm}: H_{2}^{\frac{1}{2}}(S) \rightarrow H_{2}^{-\frac{1}{2}}(S), & \chi \in X_{\varepsilon}^{3},
\end{array}
$$

are continuous.

Theorem 3.5. The operators

$$
\begin{aligned}
& \mathcal{P}_{+}: H_{2}^{0}\left(\Omega^{+}\right) \rightarrow H_{2}^{2}\left(\Omega^{+}\right), \quad \chi \in X_{\varepsilon}^{1}, \\
& \mathcal{R}_{+}: H_{2}^{0}\left(\Omega^{+}\right) \rightarrow H_{2}^{1}\left(\Omega^{+}\right), \quad \chi \in X_{\varepsilon}^{2}, \\
& \mathcal{R}_{+}: H_{2}^{1}\left(\Omega^{+}\right) \rightarrow H_{2}^{2}\left(\Omega^{+}\right), \quad \chi \in X_{\varepsilon}^{3},
\end{aligned}
$$

are continuous. 


\subsection{Auxiliary integral relations}

Let us introduce the weighted function spaces

$$
\begin{gathered}
X\left(\Omega^{-}, L\right):=\left\{w:\left(1+|x|^{2}\right)^{-\frac{1}{2}} w \in L_{2}\left(\Omega^{-}\right), \partial_{j} w \in L_{2}\left(\Omega^{-}\right),\right. \\
\left.j=1,2,3,\left(1+|x|^{2}\right)^{\frac{1}{2}} L w \in L_{2}\left(\Omega^{-}\right)\right\}, \\
Y\left(\Omega^{-}, L\right):=\left\{w:\left(1+|x|^{2}\right)^{\frac{1}{2}} w \in L_{2}\left(\Omega^{-}\right),\left(1+|x|^{2}\right)^{\frac{1}{2}} \partial_{j} w \in L_{2}\left(\Omega^{-}\right),\right. \\
\left.j=1,2,3,\left(1+|x|^{2}\right)^{\frac{1}{2}} L w \in L_{2}\left(\Omega^{-}\right)\right\} .
\end{gathered}
$$

Denote by $\ell_{0}^{+}$the extension by the zero operator from $\Omega^{+}$onto $\Omega^{-}$and by $\ell_{0}^{-}$the extension by the zero operator from $\Omega^{-}$onto $\Omega^{+}$.

Lemma 3.6. Let $u \in H^{1,0}\left(\Omega^{+}, L\right)$ and $v \in X\left(\Omega^{-}, L\right)$. Then the following relations hold:

$$
\begin{aligned}
\ell_{0}^{+} u+\mathcal{R}\left(\ell_{0}^{+} u\right) & =\mathcal{P}\left(\Delta \ell_{0}^{+}(a u)\right)-\mathcal{P}\left(\ell_{0}^{+} \Lambda(u)\right)-V\left(u^{+} \partial_{n} a\right), \\
\ell_{0}^{-} v+\mathcal{R}\left(\ell_{0}^{-} v\right) & =\mathcal{P}\left(\Delta \ell_{0}^{-}(a v)\right)-\mathcal{P}\left(\ell_{0}^{-} \Lambda(v)\right)+V\left(v^{-} \partial_{n} a\right),
\end{aligned}
$$

where $\Lambda(w):=w \Delta a+\nabla w \cdot \nabla a$ with $\nabla=\left(\partial_{1}, \partial_{2}, \partial_{3}\right)$.

Proof. Using formula (3.1), the properties of convolutions of distributions and the integration by parts formula we derive

$$
\begin{aligned}
\mathcal{R}\left(\ell_{0}^{+} u\right)= & \int_{\mathbb{R}^{3}} R(x, y) \ell_{0}^{+} u(x) d x \\
= & -\frac{1}{4 \pi a}\left\{\left[\frac{\Delta \chi(x)}{|x|}+2 \nabla \chi(x) \cdot \nabla \frac{1}{|x|}\right] * \ell_{0}^{+}(a u)\right\} \\
& -\mathcal{P}\left(\ell_{0}^{+} \Lambda(u)\right)-V\left(u^{+} \partial_{n} a\right) \\
= & -\frac{1}{4 \pi a}\left\{\left[\frac{\Delta \chi(x)}{|x|}+2 \nabla \chi(x) \cdot \nabla \frac{1}{|x|}+\chi(x) \Delta \frac{1}{|x|}\right] * \ell_{0}^{+}(a u)\right\} \\
& +\frac{1}{4 \pi a}\left[\left(\chi(x) \Delta \frac{1}{|x|}\right) * \ell_{0}^{+}(a u)\right]-\mathcal{P}\left(\ell_{0}^{+} \Lambda(u)\right)-V\left(u^{+} \partial_{n} a\right) \\
= & -\frac{1}{4 \pi a}\left[\Delta\left(\frac{\chi(x)}{|x|}\right) * \ell_{0}^{+}(a u)\right]-\frac{1}{a}\left[(\chi(x) \delta(x)) * \ell_{0}^{+}(a u)\right] \\
& -\mathcal{P}\left(\ell_{0}^{+} \Lambda(u)\right)-V\left(u^{+} \partial_{n} a\right) \\
= & \mathcal{P}\left(\Delta \ell_{0}^{+}(a u)\right)-\ell_{0}^{+} u-\mathcal{P}\left(\ell_{0}^{+} \Lambda(u)\right)-V\left(u^{+} \partial_{n} a\right) .
\end{aligned}
$$

Whence the first equality of the lemma follows. The second equality can be obtained analogously. 
Lemma 3.7. Let $u \in H^{1,0}\left(\Omega^{+}, L\right)$ and $v \in X\left(\Omega^{-}, L\right)$. Then the following relations hold:

$$
\begin{aligned}
& \mathcal{P}\left(\Delta \ell_{0}^{+}(a u)\right)=\mathcal{P}\left(\ell_{0}^{+} \Delta(a u)\right)+V\left(T^{+} u\right)+V\left(u^{+} \partial_{n} a\right)-W\left(u^{+}\right), \\
& \mathcal{P}\left(\Delta \ell_{0}^{-}(a v)\right)=\mathcal{P}\left(\ell_{0}^{-} \Delta(a v)\right)-V\left(T^{-} v\right)-V\left(v^{-} \partial_{n} a\right)+W\left(v^{-}\right) .
\end{aligned}
$$

Proof. The condition of the lemma for $u$ yields $u \in H^{1,0}\left(\Omega^{+}, \Delta\right)$. Therefore for an arbitrary $C^{\infty}$-regular test function $\varphi$ with compact support $\left(\varphi \in \mathscr{D}\left(\mathbb{R}^{3}\right)\right)$ we have Green's second formula,

$$
\int_{\Omega^{+}}(u \Delta \varphi-\varphi \Delta u) d x=\int_{S}\left(u^{+} \partial_{n} \varphi-\varphi\left[\partial_{n} u\right]^{+}\right) d S,
$$

whence

$$
\int_{\mathbb{R}^{3}}\left(\ell_{0}^{+} u\right) \Delta \varphi d x-\int_{\mathbb{R}^{3}} \varphi \ell_{0}^{+} \Delta u d x=\int_{S} u^{+} \partial_{n} \varphi d S-\int_{S}\left[\partial_{n} u\right]^{+} \varphi d S .
$$

With the help of formulas (3.6), the last equality (3.7) can be rewritten in the form

$$
\begin{array}{r}
\left\langle\Delta\left(\ell_{0}^{+} u\right), \varphi\right\rangle_{\mathbb{R}^{3}}-\left\langle\ell_{0}^{+}(\Delta u), \varphi\right\rangle_{\mathbb{R}^{3}}=-\left\langle\partial_{n}\left(u^{+} \delta_{S}\right), \varphi\right\rangle_{\mathbb{R}^{3}}-\left\langle\left[\partial_{n} u\right]^{+} \delta_{S}, \varphi\right\rangle_{\mathbb{R}^{3}}, \\
\forall \varphi \in \mathscr{D}\left(\mathbb{R}^{3}\right),
\end{array}
$$

which implies

$$
\Delta\left(\ell_{0}^{+} u\right)=\ell_{0}^{+}(\Delta u)-\partial_{n}\left(u^{+} \delta_{S}\right)-\left[\partial_{n} u\right]^{+} \delta_{S}
$$

in the distributional sense in the space of tempered distributions, $\mathcal{\rho}^{\prime}\left(\mathbb{R}^{3}\right)$. Applying the operator $\mathcal{P}$ to equation (3.8) and taking into consideration formulas (3.4) and (3.5) we get

$$
\mathcal{P}\left(\Delta\left(\ell_{0}^{+} u\right)\right)=\mathcal{P}\left(\ell_{0}^{+}(\Delta u)\right)+V\left(\left[\partial_{n} u\right]^{+}\right)-W\left(a^{-1}[u]^{+}\right) .
$$

Substituting here $a u$ for $u$ we get the first identity of the lemma. The second identity can be obtained analogously.

Further, we introduce a pseudodifferential operator $\mathcal{R}_{\mu, 0}$ with the symbol $\mu$

$$
\mu(\xi)=\mathscr{F}_{x \rightarrow \xi}\left[-\frac{1}{4 \pi}\left(\frac{\Delta \chi(x)}{|x|}+2 \nabla \chi(x) \cdot \nabla \frac{1}{|x|}\right)\right] .
$$

Simple calculations lead to the equality

$$
\begin{aligned}
\mu(\xi) & =-\frac{1}{|\xi|} \int_{0}^{\varepsilon} \partial_{\varrho}^{2} \widetilde{\chi}(\varrho) \sin (\varrho|\xi|) d \varrho \\
& =\frac{1}{|\xi|^{2}} \partial_{\varrho}^{2} \widetilde{\chi}(0)+\frac{1}{|\xi|^{2}} \int_{0}^{\varepsilon} \partial_{\varrho}^{3} \widetilde{\chi}(\varrho) \cos (\varrho|\xi|) d \varrho, \quad \xi \neq 0
\end{aligned}
$$


Whence, if $\chi \in X_{\varepsilon}^{3}$, we conclude that $\mu$ is a $C^{\infty}$-regular function in $\mathbb{R}^{3}$ decaying as $\mathcal{O}\left(|\xi|^{-2}\right)$ at infinity. Therefore, the pseudodifferential operator $\mathcal{R}_{\mu, 0}$ has order -2 , i.e., the operator

$$
\mathcal{R}_{\mu, 0}: H_{2}^{s}\left(\mathbb{R}^{3}\right) \rightarrow H_{2}^{s+2}\left(\mathbb{R}^{3}\right) \quad \forall s \in \mathbb{R}
$$

is continuous.

Lemma 3.8. Let $\chi \in X_{\epsilon}^{3}$ and $\left(1+|x|^{2}\right)^{1 / 2} g \in L_{2}\left(\mathbb{R}^{3}\right)$. Then $\left(1+|x|^{2}\right)^{1 / 2} \mathcal{M} \mathcal{R}_{\mu, 0} g$ $\in L_{2}\left(\mathbb{R}^{3}\right)$.

Proof. Due to the conditions of the lemma we have

$$
g \in L_{2}\left(\mathbb{R}^{3}\right), \quad x_{j} g \in L_{2}\left(\mathbb{R}^{3}\right), \quad j=1,2,3 .
$$

First we establish that

$$
x_{j} \mathcal{R}_{\mu, 0} g \in H_{2}^{2}\left(\mathbb{R}^{3}\right), \quad j=1,2,3 .
$$

Note that the operator $\mathcal{R}_{\mu, 0}$ is representable as

$$
\mathcal{R}_{\mu, 0} g=\mathcal{F}_{\xi \rightarrow x}^{-1}\left[\mu(\xi) \mathcal{F}_{y \rightarrow \xi} g\right],
$$

where in view of (3.9)

$$
|\mu(\xi)| \leq c(1+|\xi|)^{-2}, \quad\left|\partial_{j} \mu(\xi)\right| \leq c(1+|\xi|)^{-2}, \quad j=1,2,3,
$$

with some positive constant $c$.

Applying the Fourier transform properties we derive

$$
\begin{aligned}
x_{j} \mathcal{R}_{\mu, 0} g & =x_{j} \mathcal{F}_{\xi \rightarrow x}^{-1}\left[\mu(\xi) \mathcal{F}_{y \rightarrow \xi} g\right]=-i \mathcal{F}_{\xi \rightarrow x}^{-1}\left[\partial_{\xi_{j}}\left\{\mu(\xi) \mathcal{F}_{y \rightarrow \xi} g\right\}\right] \\
& =-i \mathcal{F}_{\xi \rightarrow x}^{-1}\left[\left\{\partial_{\xi_{j}} \mu(\xi)\right\} \mathcal{F}_{y \rightarrow \xi} g\right]-i \mathcal{F}_{\xi \rightarrow x}^{-1}\left[\mu(\xi) \partial_{\xi_{j}} \mathcal{F}_{y \rightarrow \xi} g\right] \\
& =-i \mathcal{F}_{\xi \rightarrow x}^{-1}\left[\partial_{\xi_{j}} \mu(\xi) \mathcal{F}_{y \rightarrow \xi} g\right]+\mathcal{F}_{\xi \rightarrow x}^{-1}\left[\mu(\xi) \mathcal{F}_{y \rightarrow \xi}\left(y_{j} g\right)\right] .
\end{aligned}
$$

Both summands in the last equality belong to the space $H_{2}^{2}\left(\mathbb{R}^{3}\right)$ due to the bounds (3.13) and inclusions (3.11). Thus (3.12) holds and

$$
h:=\mathcal{R}_{\mu, 0} g \in H_{2}^{2}\left(\mathbb{R}^{3}\right), \quad x_{j} h \in H_{2}^{2}\left(\mathbb{R}^{3}\right) .
$$

Further, we recall that $\mathcal{M}$ is a pseudodifferential operator of order 2 whose symbol $m(\xi)$ has the properties

$$
\begin{aligned}
c_{1}(1+|\xi|)^{2} & \leq|m(\xi)| \leq c_{2}(1+|\xi|)^{2}, \\
\left|\partial_{j} m(\xi)\right| & \leq c_{3}(1+|\xi|), \quad j=1,2,3,
\end{aligned}
$$


with some positive constants $c_{1}, c_{2}, c_{3}$. Therefore, applying the same manipulations as above we easily derive

$$
\begin{aligned}
x_{j} \mathcal{M} h & =x_{j} \mathcal{F}_{\xi \rightarrow x}^{-1}\left[m(\xi) \mathcal{F}_{y \rightarrow \xi} h\right]=-i \mathcal{F}_{\xi \rightarrow x}^{-1}\left[\partial_{\xi_{j}}\left\{m(\xi) \mathcal{F}_{y \rightarrow \xi} g\right\}\right] \\
& =-i \mathcal{F}_{\xi \rightarrow x}^{-1}\left[\left\{\partial_{\xi_{j}} m(\xi)\right\} \mathcal{F}_{y \rightarrow \xi} h\right]-i \mathcal{F}_{\xi \rightarrow x}^{-1}\left[m(\xi) \partial_{\xi_{j}} \mathcal{F}_{y \rightarrow \xi} h\right] \\
& =-i \mathcal{F}_{\xi \rightarrow x}^{-1}\left[\partial_{\xi_{j}} m(\xi) \mathcal{F}_{y \rightarrow \xi} h\right]+\mathcal{F}_{\xi \rightarrow x}^{-1}\left[m(\xi) \mathcal{F}_{y \rightarrow \xi}\left(y_{j} h\right)\right] .
\end{aligned}
$$

In view of (3.14) and (3.15) we have that $x_{j} \mathcal{M} h \in L_{2}\left(\mathbb{R}^{3}\right)$. Since $\mathcal{M} h \in L_{2}\left(\mathbb{R}^{3}\right)$, the proof follows.

\subsection{Reduction of the mixed BVP to LBDIEs}

From now on we assume that $\chi \in X_{\varepsilon}^{k}$ with $k \geq 3$.

If $u$ solves the differential equation (2.4), then from equations (3.2)-(3.3) we get

$$
\begin{aligned}
u+\mathcal{R}_{+} u-V\left(T^{+} u\right)+W\left(u^{+}\right) & =\mathcal{P}_{+} f \quad \text { in } \Omega^{+}, \\
\mathcal{R}_{+} u-V\left(T^{+} u\right)+W\left(u^{+}\right) & =\mathcal{P}_{+} f \quad \text { in } \Omega^{-}, \\
\frac{1}{2} u^{+}+\mathcal{R}_{+}^{+} u-\mathcal{V}\left(T^{+} u\right)+W\left(u^{+}\right) & =\mathcal{P}_{+}^{+} f \quad \text { on } S, \\
\frac{1}{2} T^{+} u+T^{+} \mathcal{R}_{+} u-W^{\prime}\left(T^{+} u\right)+\mathscr{L}^{+}\left(u^{+}\right) & =T^{+} \mathcal{P}_{+} f \quad \text { on } S .
\end{aligned}
$$

To get a boundary domain integral formulation of the above mixed BVP we proceed as follows.

Let $\Phi_{0} \in H_{2}^{\frac{1}{2}}(S)$ be the fixed extension of the given function $\varphi_{0}$ from the submanifold $S_{D}$ onto the whole of $S$ (see the Dirichlet condition (2.5)). An arbitrary extension $\Phi \in H_{2}^{\frac{1}{2}}(S)$ preserving the function space can be then represented as $\Phi=\Phi_{0}+\varphi$ with $\varphi \in \widetilde{H}_{2}^{\frac{1}{2}}\left(S_{N}\right)$.

Analogously, let $\Psi_{0} \in H_{2}^{-\frac{1}{2}}(S)$ be the fixed extension of the given function $\psi_{0}$ from the sub-manifold $S_{N}$ to the whole of $S$ (see the Neumann condition (2.6)). An arbitrary extension $\Psi \in H_{2}^{-\frac{1}{2}}(S)$ preserving the function space can be then represented as $\Psi=\Psi_{0}+\psi$ with $\psi \in \widetilde{H}_{2}^{-\frac{1}{2}}\left(S_{D}\right)$.

If $\varphi_{0}=0$ or $\psi_{0}=0$ then we take the canonical extension $\Phi_{0}=0$ or $\Psi_{0}=0$ on $S$.

Now, let $u$ be a solution of the mixed BVP. Evidently, we then have the equations

$$
u^{+}=\Phi_{0}+\varphi, \quad T^{+} u=\Psi_{0}+\psi,
$$

with some $\varphi \in \widetilde{H}_{2}^{\frac{1}{2}}\left(S_{N}\right)$ and $\psi \in \widetilde{H}_{2}^{-\frac{1}{2}}\left(S_{D}\right)$. 
With the help of (3.16)-(3.19) we derive the following integral relations,

$$
\begin{aligned}
u+\mathcal{R}_{+} u-V \psi+W \varphi & =F_{0} \quad \text { in } \Omega^{+}, \\
\mathcal{R}_{+} u-V \psi+W \varphi & =F_{0} \quad \text { in } \Omega^{-}, \\
r_{S_{D}} \mathcal{R}_{+}^{+} u-r_{S_{D}} \mathcal{V} \psi+r_{S_{D}} \mathcal{W} \varphi & =r_{S_{D}} F_{0}^{+}-\varphi_{0} \quad \text { on } S_{D}, \\
r_{S_{N}} T^{+} \mathcal{R}_{+} u-r_{S_{N}} \mathcal{W}^{\prime} \psi+r_{S_{N}} \mathscr{L}^{+} \varphi & =r_{S_{N}} T^{+} F_{0}-\psi_{0} \quad \text { on } S_{N},
\end{aligned}
$$

where

$$
F_{0}=\mathcal{P}_{+} f+V \Psi_{0}-W \Phi_{0} .
$$

We can consider these relations as localized boundary-domain integral equations (LBDIEs) system with respect to the unknown functions $u, \psi$, and $\varphi$.

With the help of the properties of the localized potentials and invertibility of the localized volume potential operator, we can easily show that the right-hand side functions in the equations (3.21)-(3.24) vanish if and only if $f=0, \varphi_{0}=0$, $\psi_{0}=0, \Phi_{0}=0$ and $\Psi_{0}=0$, which along with Theorem 2.1, implies, similar to proof of Theorem 3.10 below, the following equivalence of the above mixed BVP and LBDIEs system (3.21)-(3.24).

Proposition 3.9. (i) If $u$ solves the mixed $B V P$, then $(u, \psi, \varphi)$, where $\psi=$ $T^{+} u-\Psi_{0}$ and $\varphi=u^{+}-\Phi_{0}$, solves the LBDIEs system (3.21)-(3.24).

(ii) If the three vector $(u, \psi, \varphi)$ solves the LBDIEs system (3.21)-(3.24), then $u$ solves the mixed BVP and relations (3.20) hold.

(iii) The LBDIEs system (3.21)-(3.24) is uniquely solvable.

\subsection{Modified localized boundary-domain integral equations system}

In spite of Proposition 3.9, the invertibility of the matrix integral operator generated by the left-hand side expressions of system (3.21)-(3.24) is an open problem. Evidently, the choice of the right function spaces and the proof of the invertibility of the corresponding operator are very important from the point of view of stability of solutions, which is crucial for numerical applications. To overcome this problem, in what follows we modify system (3.21)-(3.24) in such a way that the new system would preserve the above mentioned equivalence to the mixed BVP and, moreover, the corresponding new operator would be invertible in appropriately chosen spaces. 
To this end, we introduce a new unknown function $v$ in $\Omega^{-}$and consider the modified system of localized boundary-domain integral equations (MLBDIE) with respect to $u, v, \psi$, and $\varphi$ :

$$
\begin{aligned}
& u+\mathcal{R}_{+} u-\mathcal{R}_{-} v-V \psi+W \varphi=F_{1} \text { in } \Omega^{+}, \\
& \mathcal{R}_{+} u-v-\mathcal{R}_{-} v-V \psi+W \varphi=F_{2} \text { in } \Omega^{-}, \\
& r_{S_{D}} \mathcal{R}_{+}^{+} u-r_{S_{D}} \mathcal{R}_{-}^{+} v-r_{S_{D}} \mathcal{V} \psi+r_{S_{D}} W_{\varphi}=F_{3} \quad \text { on } S_{D}, \\
& r_{S_{N}} T^{+} \mathcal{R}_{+} u-r_{S_{N}} T^{+} \mathcal{R}_{-} v-r_{S_{N}} \mathcal{W}^{\prime} \psi+r_{S_{N}} \mathscr{L}^{+} \varphi=F_{4} \quad \text { on } S_{N} \text {. }
\end{aligned}
$$

We assume that

$$
\begin{gathered}
u \in H_{2}^{1,0}\left(\Omega^{+}, L\right), \quad v \in X\left(\Omega^{-}, L\right), \quad \psi \in \widetilde{H}_{2}^{-\frac{1}{2}}\left(S_{D}\right), \quad \varphi \in \widetilde{H}_{2}^{\frac{1}{2}}\left(S_{N}\right), \\
F_{1} \in H_{2}^{1,0}\left(\Omega^{+}, L\right), \quad F_{2} \in Y\left(\Omega^{-}, L\right), \quad F_{3} \in H_{2}^{\frac{1}{2}}\left(S_{D}\right), \quad F_{4} \in H_{2}^{-\frac{1}{2}}\left(S_{N}\right) .
\end{gathered}
$$

We set

$$
\begin{aligned}
& \mathbb{X}:=H_{2}^{1,0}\left(\Omega^{+}, L\right) \times X\left(\Omega^{-}, L\right) \times \widetilde{H}_{2}^{-\frac{1}{2}}\left(S_{D}\right) \times \widetilde{H}_{2}^{\frac{1}{2}}\left(S_{N}\right), \\
& \mathbb{Y}:=H_{2}^{1,0}\left(\Omega^{+}, L\right) \times Y\left(\Omega^{-}, L\right) \times H_{2}^{\frac{1}{2}}\left(S_{D}\right) \times H_{2}^{-\frac{1}{2}}\left(S_{N}\right) .
\end{aligned}
$$

Evidently, $\mathbb{X}$ and $\mathbb{Y}$ are Hilbert spaces.

First we show that if

$$
\begin{array}{llll}
F_{1}=F_{0} & \text { in } \Omega^{+}, & & F_{2}=F_{0} \quad \text { in } \Omega^{-}, \\
F_{3}=r_{S_{D}} F_{0}^{+}-\varphi_{0} & \text { on } S_{D}, & F_{4}=r_{S_{N}} T^{+} F_{0}-\psi_{0} & \text { on } S_{N},
\end{array}
$$

with $F_{0}$ defined by (3.25), then the MLBDIE system (3.26)-(3.29) is equivalent to the original mixed BVP (2.4)-(2.6) in the sense described by the following assertion.

Theorem 3.10. Let conditions (3.30) be fulfilled.

(i) If $u \in H_{2}^{1}\left(\Omega^{+}\right)$solves the mixed BVP (2.4)-(2.6), then the vector function $(u, 0, \psi, \varphi)$, where

$$
\psi=T^{+} u-\Psi_{0}, \quad \varphi=u^{+}-\Phi_{0} \quad \text { on } S,
$$

solves the MLBDIE system (3.26)-(3.29).

(ii) If a vector function $(u, v, \psi, \varphi) \in \mathbb{X}$ solves the MLBDIE system (3.26)(3.29), then $u$ solves the mixed BVP (2.4)-(2.6), $v=0$ in $\Omega^{-}$, and equalities (3.31) hold. 
Proof. Let $u$ be a solution to the mixed BVP (2.4)-(2.6). It is evident that $u \in$ $H_{2}^{1,0}\left(\Omega^{+}, L\right), \psi=T^{+} u-\Psi_{0} \in \widetilde{H}_{2}^{-\frac{1}{2}}\left(S_{D}\right)$ and $\varphi=u^{+}-\Phi_{0} \in \widetilde{H}_{2}^{\frac{1}{2}}\left(S_{N}\right)$. Then from (3.2)-(3.3) it directly follows that the vector function $(u, 0, \psi, \varphi)$ solves the system (3.26)-(3.29). Thus the item (i) is proved.

Now, let a vector function $(u, v, \psi, \varphi) \in \mathbb{X}$ be a solution to system (3.26)(3.29). Taking into account relations (3.30) we can rewrite the first two equations (3.26) and (3.27) as

$$
\begin{aligned}
u+\mathcal{R}_{+} u-\mathcal{R}_{-} v-V \Psi^{*}+W \Phi^{*}=\mathcal{P}_{+} f & \text { in } \Omega^{+}, \\
\mathcal{R}_{+} u-v-\mathcal{R}_{-} v-V \Psi^{*}+W \Phi^{*}=\mathcal{P}_{+} f & \text { in } \Omega^{-},
\end{aligned}
$$

where $\Psi^{*}=\Psi_{0}+\psi$ and $\Phi^{*}=\Phi^{*}+\varphi$.

Since $u \in H_{2}^{1,0}\left(\Omega^{+}, L\right)$ we can write Green's identities (3.2)-(3.3). Taking termwise differences from (3.32)-(3.33) and (3.2)-(3.3) we arrive at the equations

$$
\begin{aligned}
\mathcal{R}_{-} v-V\left(T^{+} u-\Psi^{*}\right)+W\left(u^{+}-\Phi^{*}\right)=\mathcal{P}_{+}(L u-f) & \text { in } \Omega^{+}, \\
v+\mathcal{R}_{-} v-V\left(T^{+} u-\Psi^{*}\right)+W\left(u^{+}-\Phi^{*}\right)=\mathcal{P}_{+}(L u-f) & \text { in } \Omega^{-} .
\end{aligned}
$$

Since $v \in X\left(\Omega^{-}, L\right)$ there hold Green's identities

$$
\begin{aligned}
v+\mathcal{R}_{-} v+V\left(T^{-} v\right)-W\left(v^{-}\right)=\mathcal{P}_{-}(L v) & \text { in } \Omega^{-}, \\
\mathcal{R}_{-} v+V\left(T^{-} v\right)-W\left(v^{-}\right)=\mathcal{P}_{-}(L v) & \text { in } \Omega^{+} .
\end{aligned}
$$

Again by termwise subtraction from (3.34)-(3.35) and (3.36)-(3.37) we get

$$
\begin{aligned}
& -V\left(T^{+} u-\Psi^{*}+T^{-} v\right)+W\left(u^{+}-\Phi^{*}+v^{-}\right) \\
& \quad=\mathcal{P}_{+}(L u-f)-\mathcal{P}_{-}(L v) \text { in } \Omega^{-}, \\
& -V\left(T^{+} u-\Psi^{*}+T^{-} v\right)+W\left(u^{+}-\Phi^{*}+v^{-}\right) \\
& \quad=\mathcal{P}_{+}(L u-f)-\mathcal{P}_{-}(L v) \text { in } \Omega^{+} .
\end{aligned}
$$

Equations (3.38)-(3.39) can be rewritten as

$$
\begin{aligned}
-V\left(T^{+} u-\Psi^{*}+T^{-} v\right)+ & W\left(u^{+}-\Phi^{*}+v^{-}\right) \\
& =\mathcal{P}\left[\ell_{0}^{+}(L u-f)-\ell_{0}^{-}(L v)\right] \quad \text { in } \mathbb{R}^{3} \backslash S .
\end{aligned}
$$

Applying the jump properties of localized potential operators, we derive from (3.40)

$$
\begin{array}{rr}
u^{+}-\Phi^{*}+v^{-}=0 & \text { on } S, \\
T^{+} u-\Psi^{*}+T^{-} v=0 & \text { on } S .
\end{array}
$$


Therefore, we get from (3.40)

$$
\mathcal{P}\left[\ell_{0}^{+}(L u-f)-\ell_{0}^{-}(L v)\right]=0 \quad \text { in } \mathbb{R}^{3} .
$$

Since $\mathcal{P}=\frac{1}{a} \mathcal{P}_{0}$ is an invertible operator, we conclude that

$$
\ell_{0}^{+}(L u-f)-\ell_{0}^{-}(L v)=0 \quad \text { in } \mathbb{R}^{3},
$$

i.e.,

$$
\begin{array}{ll}
L u=f & \text { in } \Omega^{+}, \\
L v=0 & \text { in } \Omega^{-} .
\end{array}
$$

Further we derive the boundary conditions for $u$ and $v$ on $S$. From (3.26), (3.28) and (3.29) we get

$$
r_{S_{D}} u^{+}=\varphi_{0} \quad \text { on } S_{D}, \quad r_{S_{N}} T^{+} u=\psi_{0} \quad \text { on } S_{N} .
$$

Consequently, from (3.41) and (3.42) we conclude

$$
r_{S_{D}} v^{-}=0 \quad \text { on } S_{D}, \quad r_{S_{N}} T^{-} v=0 \quad \text { on } S_{N} .
$$

Thus, from (3.43) and (3.45) it follows that $u$ solves the mixed BVP (2.4)-(2.6), while (3.44) and (3.46) show that $v$ solves the homogeneous mixed exterior BVP in $\Omega^{-}$. Since $v \in X\left(\Omega^{-}, L\right)$ we can write Green's formula in $\Omega^{-}$,

$$
\int_{\Omega^{-}}[v L v+E(v, v)] d x=-\left\langle T^{-} v, v^{-}\right\rangle_{S}
$$

which implies that $v=0$ in $\Omega^{-}$. As a consequence, from (3.41) and (3.42), equalities (3.31) follow. This completes the proof.

Further, we show the following uniqueness result.

Theorem 3.11. The homogeneous MLBDIEs system possesses only a trivial solution.

Proof. First let us recall that the right-hand side functions $F_{j}, j=\overline{1,4}$, given by (3.30) vanish if and only if $f=0$ in $\Omega^{+}, \Psi_{0}=\Phi_{0}=0$ on $S, \varphi_{0}=0$ on $S_{D}$, and $\psi_{0}=0$ on $S_{N}$.

Further, let $(u, v, \psi, \varphi) \in \mathbb{X}$ be a solution to the homogeneous MLBDIEs system. Then Theorem 3.10(ii) yields that $u$ solves the homogeneous mixed BVP, relations (3.31) hold and $v=0$ in $\Omega^{-}$. Consequently, $u$ vanishes in $\Omega^{+}$due to the uniqueness Theorem 2.1(i), $\varphi=0$ and $\psi=0$ on $S$ in view of (3.31). 


\subsection{Existence results for the MLBDIEs system}

Let us introduce the matrix operator generated by the left-hand side expressions of the MLBDIEs system (3.26)-(3.29)

$$
\mathcal{N}:=\left[\begin{array}{llll}
r_{\Omega^{+}}\left(I+\mathcal{R}_{+}\right) & -r_{\Omega^{+}} \mathcal{R}_{-} & -r_{\Omega^{+}} V & r_{\Omega^{+}} W \\
r_{\Omega^{-}} \mathcal{R}_{+} & -r_{\Omega^{-}}\left(I+\mathcal{R}_{-}\right) & -r_{\Omega^{-}} V & r_{\Omega^{-}} W \\
r_{S_{D}} \mathcal{R}_{+}^{+} & -r_{S_{D}} \mathcal{R}_{-}^{+} & -r_{S_{D}} \mathcal{V} & r_{S_{D}} \mathcal{W} \\
r_{S_{N}} T^{+} \mathcal{R}_{+} & -r_{S_{N}} T^{+} \mathcal{R}_{-} & -r_{S_{N}} \mathcal{W}^{\prime} & r_{S_{N}} \mathscr{L}^{+}
\end{array}\right] .
$$

First we show the following assertion.

Theorem 3.12. The operator

$$
\mathcal{N}: \mathbb{X} \rightarrow \mathbb{Y}
$$

is continuous.

Proof. Due to the properties of the localized potentials (see Theorems 3.2 and 3.5), it suffices to prove that $v+\mathcal{R}_{-} v \in Y\left(\Omega^{-}, L\right)$ and $r_{\Omega^{+}} \mathcal{R}_{-} v \in H^{1,0}\left(\Omega^{+}\right)$for $v \in X\left(\Omega^{-}, L\right)$, i.e.,

$$
\begin{aligned}
r_{\Omega^{-}}\left(I+\mathcal{R}_{-}\right): X\left(\Omega^{-}, L\right) & \rightarrow Y\left(\Omega^{-}, L\right), \\
r_{\Omega^{+}} \mathcal{R}_{-}: X\left(\Omega^{-}, L\right) & \rightarrow H^{1,0}\left(\Omega^{+}, L\right) .
\end{aligned}
$$

By Green's formulas (3.36) and (3.37) we have

$$
\begin{aligned}
& v+\mathcal{R}_{-} v=\mathcal{P}_{-}(L v)-V\left(T^{-} v\right)+W\left(v^{-}\right) \text {in } \Omega^{-}, \\
& \mathcal{R}_{-} v=\mathcal{P}_{-}(L v)-V\left(T^{-} v\right)+W\left(v^{-}\right) \text {in } \Omega^{+} .
\end{aligned}
$$

Evidently, $V\left(T^{-} v\right)$ and $W\left(v^{-}\right)$have compact supports and belong to the space $Y\left(\Omega^{-}, L\right)$. Let us investigate the localized volume potential

$$
\mathcal{P}_{-}(L v)=\mathcal{P}_{0}^{-}(L v) \quad \text { in } \mathbb{R}^{3} .
$$

Clearly, $\mathcal{P} \ell_{0}^{-}(L v) \in H_{2}^{2}\left(\mathbb{R}^{3}\right)$ since $\mathcal{P}$ is a pseudodifferential operator of order -2 and $\ell_{0}^{-}(L v) \in L_{2}\left(\mathbb{R}^{3}\right)$. Set $g=\ell_{0}^{-}(L v)$. Then $v \in X\left(\Omega^{-}, L\right)$ yields

$$
g \in L_{2}\left(\mathbb{R}^{3}\right), \quad x_{j} g \in L_{2}\left(\mathbb{R}^{3}\right) .
$$

Next we establish that

$$
x_{j} \mathcal{P} g \in H_{2}^{2}\left(\mathbb{R}^{3}\right), \quad j=1,2,3 .
$$


Note that the operator $\mathcal{P}_{0}=a \mathcal{P}$ as a pseudodifferential operator is representable as

$$
\mathcal{P}_{0} g=\mathcal{F}_{\xi \rightarrow x}^{-1}\left[\lambda(\xi) \mathcal{F}_{y \rightarrow \xi} g\right],
$$

where the symbol $\lambda(\xi)$ has the properties (for details see [4])

$$
\begin{aligned}
c_{1}(1+|\xi|)^{-2} & \leq|\lambda(\xi)| \leq c_{2}(1+|\xi|)^{-2}, \\
\left|\partial_{j} \lambda(\xi)\right| & \leq c_{3}(1+|\xi|)^{-3}, \quad j=1,2,3,
\end{aligned}
$$

with some positive constants $c_{1}, c_{2}, c_{3}$.

Applying the Fourier transform properties we derive

$$
\begin{aligned}
x_{j} \mathcal{P}_{0} g & =x_{j} \mathscr{F}_{\xi \rightarrow x}^{-1}\left[\lambda(\xi) \mathscr{F}_{y \rightarrow \xi} g\right]=-i \mathscr{F}_{\xi \rightarrow x}^{-1}\left[\partial_{\xi_{j}}\left\{\lambda(\xi) \mathscr{F}_{y \rightarrow \xi} g\right\}\right] \\
& =-i \mathcal{F}_{\xi \rightarrow x}^{-1}\left[\left\{\partial_{\xi_{j}} \lambda(\xi)\right\} \mathscr{F}_{y \rightarrow \xi} g\right]-i \mathcal{F}_{\xi \rightarrow x}^{-1}\left[\lambda(\xi) \partial_{\xi_{j}} \mathcal{F}_{y \rightarrow \xi} g\right] \\
& =-i \mathscr{F}_{\xi \rightarrow x}^{-1}\left[\partial_{\xi_{j}} \lambda(\xi) \mathscr{F}_{y \rightarrow \xi} g\right]+\mathscr{F}_{\xi \rightarrow x}^{-1}\left[\lambda(\xi) \mathscr{F}_{y \rightarrow \xi}\left(y_{j} g\right)\right] .
\end{aligned}
$$

The first summand in the last equality belongs to the space $H_{2}^{3}\left(\mathbb{R}^{3}\right)$, while the second one belongs to $H_{2}^{2}\left(\mathbb{R}^{3}\right)$ due to bounds (3.53) and inclusions (3.51). Thus (3.52) holds.

From the above inclusions and (3.49) it follows that

$$
\begin{aligned}
\left(1+|x|^{2}\right)^{1 / 2}\left(v+\mathcal{R}_{-} v\right) & \in L_{2}\left(\Omega^{-}\right), \\
\left(1+|x|^{2}\right)^{1 / 2} \partial_{j}\left(v+\mathcal{R}_{-} v\right) & \in L_{2}\left(\Omega^{-}\right), \quad j=1,2,3, \\
\left(1+|x|^{2}\right)^{1 / 2} L\left(v+\mathcal{R}_{-} v\right) & \in L_{2}\left(\Omega^{-}\right),
\end{aligned}
$$

i.e., $v+\mathcal{R}_{-} v \in Y\left(\Omega^{-}, L\right)$. Since $Y\left(\Omega^{-}, L\right) \subset X\left(\Omega^{-}, L\right)$, as a consequence we conclude that $\mathcal{R}_{-}: X\left(\Omega^{-}, L\right) \rightarrow X\left(\Omega^{-}, L\right)$.

The second mapping property in (3.48) immediately follows from (3.50).

Next we prove the following basic invertibility result for operator (3.47).

Theorem 3.13. The operator

$$
\mathcal{N}: \mathbb{X} \rightarrow \mathbb{Y}
$$

is invertible.

Proof. From Theorem 3.11 it follows that operator (3.54) is injective. It remains to show that the operator (3.54) is surjective, that is, the nonhomogeneous MLBDIEs system (3.26)-(3.29), i.e., the vector equation

$$
\mathcal{N} U=F
$$

with $U=(u, v, \psi, \varphi)^{\top} \in \mathbb{X}$ is solvable for arbitrary $F=\left(F_{1}, F_{2}, F_{3}, F_{4}\right)^{\top} \in \mathbb{Y}$. 
To this end, first we reduce equation (3.55) to the equivalent, uniquely solvable two coupled mixed BVPs.

To derive the corresponding differential equations and boundary conditions we rewrite the first two equations (3.26)-(3.27) in the form

$$
\begin{aligned}
\left(u-F_{1}\right)+\mathcal{R}_{+}\left(u-F_{1}\right)-\mathcal{R}_{-}\left(v+F_{2}\right)-V \psi & +W \varphi \\
& =-\mathcal{R}_{+} F_{1}-\mathcal{R}_{-} F_{2} \text { in } \Omega^{+}, \\
\mathcal{R}_{+}\left(u-F_{1}\right)-\left(v+F_{2}\right)-\mathcal{R}_{-}\left(v+F_{2}\right)-V \psi & +W \varphi \\
& =-\mathcal{R}_{+} F_{1}-\mathcal{R}_{-} F_{2} \text { in } \Omega^{-},
\end{aligned}
$$

i.e.,

$$
\begin{array}{r}
\ell_{0}^{+}\left(u-F_{1}\right)+\mathcal{R} \ell_{0}^{+}\left(u-F_{1}\right)-\ell_{0}^{-}\left(v+F_{2}\right)-\mathcal{R} \ell_{0}^{-}\left(v+F_{2}\right)-V \psi+W \varphi \\
=-\mathcal{R} \ell_{0}^{+} F_{1}-\mathcal{R} \ell_{0}^{-} F_{2} \text { in } \mathbb{R}^{3} \backslash S .
\end{array}
$$

We have the formulas (see Lemma 3.6)

$$
\begin{aligned}
\ell_{0}^{+}\left(u-F_{1}\right)+ & \mathcal{R} \ell_{0}^{+}\left(u-F_{1}\right)= \\
& \mathcal{P}\left(\Delta \ell_{0}^{+}\left[a\left(u-F_{1}\right)\right]\right)-\mathcal{P}\left(\ell_{0}^{+} \Lambda\left(u-F_{1}\right)\right) \\
& -V\left(\left(u-F_{1}\right)^{+} \partial_{n} a\right), \\
\ell_{0}^{-}\left(v+F_{2}\right)+\mathcal{R} \ell_{0}^{-}\left(v+F_{2}\right)= & \mathcal{P}\left(\Delta \ell_{0}^{-}\left[a\left(v+F_{2}\right)\right]\right)-\mathcal{P}\left(\ell_{0}^{-} \Lambda\left(v+F_{2}\right)\right) \\
& +V\left(\left(v+F_{2}\right)^{-} \partial_{n} a\right) .
\end{aligned}
$$

Moreover, as in the proof of Lemma 3.6, it immediately follows that

$$
\begin{aligned}
& \mathcal{R} \ell_{0}^{+} F_{1}=\mathcal{R}_{\mu}\left(\ell_{0}^{+}\left(a F_{1}\right)\right)-\mathcal{P}\left(\ell_{0}^{+} \Lambda\left(F_{1}\right)\right)-V\left(F_{1}^{+} \partial_{n} a\right), \\
& \mathcal{R} \ell_{0}^{-} F_{2}=\mathcal{R}_{\mu}\left(\ell_{0}^{-}\left(a F_{2}\right)\right)-\mathcal{P}\left(\ell_{0}^{-} \Lambda\left(F_{2}\right)\right)+V\left(F_{2}^{-} \partial_{n} a\right),
\end{aligned}
$$

where $a \mathcal{R}_{\mu}=\mathcal{R}_{\mu, 0}$, and $\mathcal{R}_{\mu, 0}$ is a pseudodifferential operator of order -2 with the symbol $\mu(\xi)$ (see Subsection 3.3).

With the help of the equalities (3.57)-(3.60) we get from (3.56)

$$
\begin{aligned}
\mathcal{P}( & \left.\Delta \ell_{0}^{+}\left[a\left(u-F_{1}\right)\right]\right)-\mathcal{P}\left(\ell_{0}^{+} \Lambda(u)\right)-V\left(u^{+} \partial_{n} a\right) \\
& -\mathcal{P}\left(\Delta \ell_{0}^{-}\left[a\left(v+F_{2}\right)\right]\right)+\mathcal{P}\left(\ell_{0}^{-} \Lambda(v)\right)-V\left(v^{-} \partial_{n} a\right)-V(\psi)+W(\varphi) \\
= & -\mathcal{R}_{\mu}\left(\ell_{0}^{+}\left(a F_{1}\right)\right)-\mathcal{R}_{\mu}\left(\ell_{0}^{-}\left(a F_{2}\right)\right) \text { in } \mathbb{R}^{3} \backslash S .
\end{aligned}
$$


In accordance with the formulas (see Lemma 3.7)

$$
\begin{aligned}
\mathcal{P}\left(\Delta \ell_{0}^{+}\left[a\left(u-F_{1}\right)\right]\right)= & \mathcal{P}\left(\ell_{0}^{+} \Delta\left[a\left(u-F_{1}\right)\right]\right)+V\left(T^{+} u-T^{+} F_{1}\right) \\
& +V\left(\left[u^{+}-F_{1}^{+}\right] \partial_{n} a\right)-W\left(u^{+}-F_{1}^{+}\right), \\
\mathcal{P}\left(\Delta \ell_{0}^{-}\left(a\left(v+F_{2}\right)\right)\right)= & \mathcal{P}\left(\ell_{0}^{-} \Delta\left[a\left(v+F_{2}\right)\right]\right)-V\left(T^{-} v+T^{-} F_{2}\right) \\
& -V\left(\left(v^{-}+F_{2}^{-}\right) \partial_{n} a\right)+W\left(v^{-}+F_{2}^{-}\right),
\end{aligned}
$$

we get from (3.61)

$$
\begin{aligned}
\mathcal{P}\left(\ell_{0}^{+}\right. & \left.\Delta\left[a\left(u-F_{1}\right)\right]\right)+V\left(T^{+} u-T^{+} F_{1}\right)+V\left(\left(u^{+}-F_{1}^{+}\right) \partial_{n} a\right) \\
& -W\left(u^{+}-F_{1}^{+}\right)-\mathcal{P}\left(\ell_{0}^{+} \Lambda(u)\right)-V\left(u^{+} \partial_{n} a\right)-\mathcal{P}\left(\ell_{0}^{-} \Delta\left[a\left(v+F_{2}\right)\right]\right) \\
& +V\left(T^{-} v+T^{-} F_{2}\right)+V\left(\left(v^{-}+F_{2}^{-}\right) \partial_{n} a\right)-W\left(v^{-}+F_{2}^{-}\right) \\
& +\mathcal{P}\left(\ell_{0}^{-} \Lambda(v)\right)-V\left(v^{-} \partial_{n} a\right)-V(\psi)+W(\varphi) \\
= & -\mathcal{R}_{\mu}\left(\ell_{0}^{+}\left(a F_{1}\right)\right)-\mathcal{R}_{\mu}\left(\ell_{0}^{-}\left(a F_{2}\right)\right) \quad \text { in } \mathbb{R}^{3} \backslash S .
\end{aligned}
$$

Whence, using the notation

$$
\begin{aligned}
& \widetilde{\Psi}=T^{+} u-T^{+} F_{1}-F_{1}^{+} \partial_{n} a+T^{-} v+T^{-} F_{2}+F_{2}^{-} \partial_{n} a-\psi, \\
& \widetilde{\Phi}=u^{+}-F_{1}^{+}+v^{-}+F_{2}^{-}-\varphi,
\end{aligned}
$$

we get

$$
\begin{aligned}
\mathcal{P}\left(\ell_{0}^{+} \Delta\left[a\left(u-F_{1}\right)\right]\right)+V \widetilde{\Psi}-W \widetilde{\Phi}-\mathcal{P}\left(\ell_{0}^{+} \Lambda(u)\right)-\mathcal{P}\left(\ell_{0}^{-} \Delta\left[a\left(v+F_{2}\right)\right]\right) \\
+\mathcal{P}\left(\ell_{0}^{-} \Lambda(v)\right)=-\mathcal{R}_{\mu}\left(\ell_{0}^{+}\left(a F_{1}\right)\right)-\mathcal{R}_{\mu}\left(\ell_{0}^{-}\left(a F_{2}\right)\right) \quad \text { in } \mathbb{R}^{3} \backslash S
\end{aligned}
$$

Taking the difference of interior and exterior traces on $S$, from this equation we obtain $\widetilde{\Phi}=0$ on $S$. Further, the difference of interior and exterior co-normal differentiation operators, $T^{ \pm}$, of the same equation gives $\widetilde{\Psi}=0$ on $S$. Thus

$$
\begin{aligned}
& \psi=T^{+} u-T^{+} F_{1}-F_{1}^{+} \partial_{n} a+T^{-} v+T^{-} F_{2}+F_{2}^{-} \partial_{n} a, \\
& \varphi=u^{+}-F_{1}^{+}+v^{-}+F_{2}^{-} .
\end{aligned}
$$

In view of these equalities, multiplying (3.64) by $a$ and recalling that $\mathcal{P}_{0}=a \mathcal{P}$, we get

$$
\begin{aligned}
\mathcal{P}_{0}\left(\ell_{0}^{+} \Delta\left[a\left(u-F_{1}\right)\right]\right) & -\mathcal{P}_{0}\left(\ell_{0}^{+} \Lambda(u)\right)-\mathcal{P}_{0}\left(\ell_{0}^{-} \Delta\left[a\left(v+F_{2}\right)\right]\right)+\mathcal{P}_{0}\left(\ell_{0}^{-} \Lambda(v)\right) \\
& =-\mathcal{R}_{\mu, 0}\left(\ell_{0}^{+}\left(a F_{1}\right)\right)-\mathcal{R}_{\mu, 0}\left(\ell_{0}^{-}\left(a F_{2}\right)\right) \quad \text { in } \mathbb{R}^{3} .
\end{aligned}
$$


Since the pseudodifferential operator $\mathcal{M}$ is inverse to $\mathcal{P}_{0}$, from (3.67) we derive

$$
\begin{aligned}
\ell_{0}^{+} \Delta\left[a\left(u-F_{1}\right)\right]-\ell_{0}^{+} \Lambda(u) & -\ell_{0}^{-} \Delta\left[a\left(v+F_{2}\right)\right]+\ell_{0}^{-} \Lambda(v) \\
& =-\mathcal{M} \mathcal{R}_{\mu, 0}\left[\ell_{0}^{+}\left(a F_{1}\right)+\ell_{0}^{-}\left(a F_{2}\right)\right] \text { in } \mathbb{R}^{3} .
\end{aligned}
$$

Note that

$$
\Delta(a w)=L w+\Lambda(w) .
$$

Therefore we can rewrite (3.68) as

$$
\begin{aligned}
\ell_{0}^{+} L u-\ell_{0}^{-} L v= & \ell_{0}^{+} L F_{1}+\ell_{0}^{-} L F_{2}+\ell_{0}^{+} \Lambda\left(F_{1}\right)+\ell_{0}^{-} \Lambda\left(F_{2}\right) \\
& -\mathcal{M} \mathcal{R}_{\mu, 0}\left[\ell_{0}^{+}\left(a F_{1}\right)+\ell_{0}^{-}\left(a F_{2}\right)\right] \text { in } \mathbb{R}^{3},
\end{aligned}
$$

whence taking restrictions on $\Omega^{+}$and $\Omega^{-}$we finally arrive at the differential equations

$$
\begin{aligned}
& L u=G \text { with } \\
& G=L F_{1}+\Lambda\left(F_{1}\right)-r_{\Omega^{+}} \mathcal{M} \mathcal{R}_{\mu, 0}\left[\ell_{0}^{+}\left(a F_{1}\right)+\ell_{0}^{-}\left(a F_{2}\right)\right] \text { in } \Omega^{+}, \\
& L v=Q \text { with } \\
& Q=-L F_{2}-\Lambda\left(F_{2}\right)+r_{\Omega^{-}} \mathcal{M} \mathcal{R}_{\mu, 0}\left[\ell_{0}^{+}\left(a F_{1}\right)+\ell_{0}^{-}\left(a F_{2}\right)\right] \text { in } \Omega^{-} .
\end{aligned}
$$

Further, from the first, third and fourth equations of the MLBDIEs system (3.26)(3.29) we obtain the boundary conditions

$$
\begin{aligned}
& r_{S_{D}} u^{+}=g_{1} \quad \text { with } g_{1}=r_{S_{D}} F_{1}^{+}-F_{3} \text { on } S_{D}, \\
& r_{S_{N}} T^{+} u=g_{2} \quad \text { with } g_{2}=r_{S_{N}} T^{+} F_{1}-F_{4} \text { on } S_{N} \text {. }
\end{aligned}
$$

With the help of equalities (3.65)-(3.66) and (3.72)-(3.73) we have

$$
\begin{aligned}
r_{S_{D}} v^{-}=q_{1} \quad \text { with } q_{1}= & -g_{1}+r_{S_{D}} F_{1}^{+}-r_{S_{D}} F_{2}^{-} \text {on } S_{D}, \\
r_{S_{N}} T^{-} v=q_{2} \quad \text { with } q_{2}= & -g_{2}+r_{S_{N}} T^{+} F_{1}-r_{S_{N}} T^{-} F_{2} \\
& +r_{S_{N}}\left(F_{1}^{+}-F_{2}^{-}\right) \partial_{n} a \text { on } S_{N} .
\end{aligned}
$$

Thus we have proved that if $(u, v, \psi, \varphi)^{\top}$ solves MLBDIEs (3.26)-(3.29), then relations (3.65)-(3.66) hold, and $u$ and $v$ are solutions of the following mixed 
BVPs:

$$
\begin{array}{cl}
L u=G \text { in } \Omega^{+}, & G \in L_{2}\left(\Omega^{+}\right), \\
r_{S_{D}} u^{+}=g_{1} \text { on } S_{D}, & g_{1} \in H_{2}^{\frac{1}{2}}\left(S_{D}\right), \\
r_{S_{N}} T^{+} u=g_{2} \text { on } S_{N}, & g_{2} \in H_{2}^{-\frac{1}{2}}\left(S_{N}\right),
\end{array}
$$

and

$$
\begin{array}{rlrl}
L v & =Q \text { in } \Omega^{-}, & & \left(1+|x|^{2}\right)^{1 / 2} Q \in L_{2}\left(\Omega^{-}\right), \\
r_{S_{D}} v^{-} & =q_{1} \text { on } S_{D}, & & q_{1} \in H_{2}^{\frac{1}{2}}\left(S_{D}\right), \\
r_{S_{N}} T^{-} v & =q_{2} \text { on } S_{N}, \quad & q_{2} \in H_{2}^{-\frac{1}{2}}\left(S_{N}\right) .
\end{array}
$$

Inclusion (3.79) for $Q$ follows from Lemma 3.8.

Now we show the inverse. Let $\left(F_{1}, F_{2}, F_{3}, F_{4}\right)^{\top} \in \mathbb{Y}$ be an arbitrary vector function and $u \in H_{2}^{1,0}\left(\Omega^{+}, L\right)$ and $v \in X\left(\Omega^{-}, L\right)$ be solutions to the mixed BVPs (3.76)-(3.78) and (3.79)-(3.81) with the given functions $G, g_{1}, g_{2}, Q$, $q_{1}, q_{2}$ defined by the above equalities (3.70)-(3.75). Further, construct the functions $\psi \in \widetilde{H}^{-\frac{1}{2}}\left(S_{D}\right)$ and $\varphi \in \widetilde{H}^{\frac{1}{2}}\left(S_{N}\right)$ by equalities (3.65)-(3.66). In what follows we prove that the vector function $(u, v, \psi, \varphi)^{\top} \in \mathbb{X}$ solves MLBDIEs (3.26)(3.29).

Rewrite equations (3.76) and (3.79) as

$$
\begin{aligned}
& L\left(u-F_{1}\right)-\Lambda\left(F_{1}\right)=-r_{\Omega^{+}} \mathcal{M} \mathcal{R}_{\mu, 0}\left[\ell_{0}^{+}\left(a F_{1}\right)+\ell_{0}^{-}\left(a F_{2}\right)\right] \text { in } \Omega^{+}, \\
& L\left(v+F_{2}\right)+\Lambda\left(F_{2}\right)=r_{\Omega^{-}} \mathcal{M} \mathcal{R}_{\mu, 0}\left[\ell_{0}^{+}\left(a F_{1}\right)+\ell_{0}^{-}\left(a F_{2}\right)\right] \text { in } \Omega^{-} .
\end{aligned}
$$

With the help of identity (3.69) we get

$$
\begin{aligned}
& \Delta\left[a\left(u-F_{1}\right)\right]-\Lambda(u)=-r_{\Omega^{+}} \mathcal{M} \mathcal{R}_{\mu, 0}\left[\ell_{0}^{+}\left(a F_{1}\right)+\ell_{0}^{-}\left(a F_{2}\right)\right] \text { in } \Omega^{+}, \\
& -\Delta\left[a\left(v+F_{2}\right)\right]+\Lambda(v)=-r_{\Omega^{-}} \mathcal{M} \mathcal{R}_{\mu, 0}\left[\ell_{0}^{+}\left(a F_{1}\right)+\ell_{0}^{-}\left(a F_{2}\right)\right] \text { in } \Omega^{-},
\end{aligned}
$$

whence employing the extension by zero operators $\ell_{0}^{ \pm}$we can write

$$
\begin{aligned}
& \ell_{0}^{+} \Delta\left[a\left(u-F_{1}\right)\right]-\ell_{0}^{+} \Lambda(u)=-\ell_{0}^{+} \mathcal{M} \mathcal{R}_{\mu, 0}\left[\ell_{0}^{+}\left(a F_{1}\right)+\ell_{0}^{-}\left(a F_{2}\right)\right] \text { in } \Omega^{+}, \\
& -\ell_{0}^{-} \Delta\left[a\left(v+F_{2}\right)\right]+\ell_{0}^{-} \Lambda(v)=-\ell_{0}^{-} \mathcal{M} \mathcal{R}_{\mu, 0}\left[\ell_{0}^{+}\left(a F_{1}\right)+\ell_{0}^{-}\left(a F_{2}\right)\right] \text { in } \Omega^{-} \text {. }
\end{aligned}
$$

We can rewrite these equations as

$$
\begin{aligned}
\ell_{0}^{+} \Delta\left[a\left(u-F_{1}\right)\right]-\ell_{0}^{+} \Lambda(u)-\ell_{0}^{-} & \Delta\left[a\left(v+F_{2}\right)\right]+\ell_{0}^{-} \Lambda(v) \\
& =-\mathcal{M} \mathcal{R}_{\mu, 0}\left[\ell_{0}^{+}\left(a F_{1}\right)+\ell_{0}^{-}\left(a F_{2}\right)\right] \text { in } \mathbb{R}^{3} .
\end{aligned}
$$

since the right-hand side is an $L_{2}$ function in $\mathbb{R}^{3}$. 
Apply the operator $\mathcal{P}=\frac{1}{a} \mathcal{P}_{0}$ to this equation and take into consideration that $\mathcal{P}_{0}$ is the inverse to $\mathcal{M}$

$$
\begin{array}{r}
\mathcal{P}\left(\ell_{0}^{+} \Delta\left[a\left(u-F_{1}\right)\right]\right)-\mathcal{P}\left(\ell_{0}^{+} \Lambda(u)\right)-\mathcal{P}\left(\ell_{0}^{-} \Delta\left[a\left(v+F_{2}\right)\right]\right)+\mathcal{P}\left(\ell_{0}^{-} \Lambda(v)\right) \\
=-\mathcal{R}_{\mu}\left[\ell_{0}^{+}\left(a F_{1}\right)+\ell_{0}^{-}\left(a F_{2}\right)\right] \quad \text { in } \mathbb{R}^{3} .
\end{array}
$$

Determine $\mathcal{P}\left(\ell_{0}^{+} \Delta\left[a\left(u-F_{1}\right)\right]\right)$ and $\mathcal{P}\left(\ell_{0}^{-} \Delta\left[a\left(v+F_{2}\right)\right]\right)$ from formulas (3.62)(3.63) and substitute into (3.82)

$$
\begin{aligned}
\mathcal{P} & \left(\Delta \ell_{0}^{+}\left[a\left(u-F_{1}\right)\right]\right)-V\left(T^{+} u-T^{+} F_{1}\right)-V\left(\left(u^{+}-F_{1}^{+}\right) \partial_{n} a\right) \\
& +W\left(u^{+}-F_{1}^{+}\right)-\mathcal{P}\left(\ell_{0}^{+} \Lambda(u)\right)-\mathcal{P}\left(\Delta \ell_{0}^{-}\left[a\left(v+F_{2}\right)\right]\right)-V\left(T^{-} v+T^{-} F_{2}\right) \\
& -V\left(\left(v^{-}+F_{2}^{-}\right) \partial_{n} a\right)+W\left(v^{-}+F_{2}^{-}\right)+\mathcal{P}\left(\ell_{0}^{-} \Lambda(v)\right) \\
& =-\mathcal{R}_{\mu}\left(\ell_{0}^{+}\left(a F_{1}\right)\right)-\mathcal{R}_{\mu}\left(\ell_{0}^{-}\left(a F_{2}\right)\right) \text { in } \mathbb{R}^{3} .
\end{aligned}
$$

In view of equalities (3.65)-(3.66) it is easy to check that in (3.83) the densities of the single and double layer potentials are $-\psi-u^{+} \partial_{n} a-v^{-} \partial_{n} a$ and $\varphi$, respectively. Therefore we arrive at the equation

$$
\begin{aligned}
\mathcal{P}\left(\Delta \ell_{0}^{+}\left[a\left(u-F_{1}\right)\right]\right)-\mathcal{P}\left(\ell_{0}^{+} \Lambda(u)\right)-V\left(u^{+} \partial_{n} a\right) & \\
& -\mathcal{P}\left(\Delta \ell_{0}^{-}\left[a\left(v+F_{2}\right)\right]\right)+\mathcal{P}\left(\ell_{0}^{-} \Lambda(v)\right)-V\left(v^{-} \partial_{n} a\right)-V \psi+W \varphi \\
= & -\mathcal{R}_{\mu}\left(\ell_{0}^{+}\left(a F_{1}\right)\right)-\mathcal{R}_{\mu}\left(\ell_{0}^{-}\left(a F_{2}\right)\right) \text { in } \mathbb{R}^{3} .
\end{aligned}
$$

Add to both sides of the equation the expression

$$
\mathcal{P}\left(\ell_{0}^{+} \Lambda\left(F_{1}\right)\right)+V\left(F_{1}^{+} \partial_{n} a\right)+\mathcal{P}\left(\ell_{0}^{-} \Lambda\left(F_{2}\right)\right)-V\left(F_{2}^{-} \partial_{n} a\right)
$$

to obtain

$$
\begin{aligned}
\mathcal{P}\left(\Delta \ell_{0}^{+}\left[a\left(u-F_{1}\right)\right]\right)-\mathcal{P}\left(\ell_{0}^{+} \Lambda\left(u-F_{1}\right)\right)-V\left(\left(u^{+}-F_{1}^{+}\right) \partial_{n} a\right) \\
-\mathcal{P}\left(\Delta \ell_{0}^{-}\left[a\left(v+F_{2}\right)\right]\right)+\mathcal{P}\left(\ell_{0}^{-} \Lambda\left(v+F_{2}\right)\right)-V\left(\left(v^{-}+F_{2}^{-}\right) \partial_{n} a\right)-V \psi+W \varphi \\
=-\mathcal{R}_{\mu}\left(\ell_{0}^{+}\left(a F_{1}\right)\right)+\mathcal{P}\left(\ell_{0}^{+} \Lambda\left(F_{1}\right)\right)+V\left(F_{1}^{+} \partial_{n} a\right) \\
\quad-\mathcal{R}_{\mu}\left(\ell_{0}^{-}\left(a F_{2}\right)\right)+\mathcal{P}\left(\ell_{0}^{-} \Lambda\left(F_{2}\right)\right)-V\left(F_{2}^{-} \partial_{n} a\right) \text { in } \mathbb{R}^{3} .
\end{aligned}
$$

From this equation with the help of (3.57)-(3.60) we get

$$
\begin{aligned}
\ell_{0}^{+}\left(u-F_{1}\right)+\mathcal{R} \ell_{0}^{+}\left(u-F_{1}\right)-\ell_{0}^{-}\left(v+F_{2}\right) & -\mathcal{R} \ell_{0}^{-}\left(v+F_{2}\right)-V \psi+W \varphi \\
& =-\mathcal{R} \ell_{0}^{+} F_{1}-\mathcal{R} \ell_{0}^{-} F_{2} \text { in } \mathbb{R}^{3} .
\end{aligned}
$$


Restrictions of this equation on $\Omega^{+}$and $\Omega^{-}$give

$$
\begin{array}{cl}
u+\mathcal{R}_{+} u-\mathcal{R}_{-} v-V \psi+W \varphi=F_{1} & \text { in } \Omega^{+}, \\
\mathcal{R}_{+} u-v-\mathcal{R}_{-} v-V \psi+W \varphi=F_{2} & \text { in } \Omega^{-},
\end{array}
$$

which coincide with the first two equations in the MLBDIEs system (3.26)-(3.29). Now from equations (3.72), (3.77) and (3.85) it follows that

$$
\begin{aligned}
& r_{S_{D}} u^{+}=r_{S_{D}} F_{1}^{+}-\left(r_{S_{D}} \mathcal{R}_{+}^{+} u-r_{S_{D}} \mathcal{R}_{-}^{+} v-r_{S_{D}} \mathcal{V} \psi+r_{S_{D}} \mathcal{W}_{\varphi}\right), \\
& r_{S_{D}} u^{+}=r_{S_{D}} F_{1}^{+}-F_{3},
\end{aligned}
$$

which coincides with the third equation in the MLBDIEs system (3.26)-(3.29),

$$
r_{S_{D}} \mathcal{R}_{+}^{+} u-r_{S_{D}} \mathcal{R}_{-}^{+} v-r_{S_{D}} \mathcal{V} \psi+r_{S_{D}} W_{\varphi}=F_{3} \quad \text { on } S_{D} .
$$

Analogously, from equations (3.73), (3.78) and (3.85) we get

$r_{S_{N}} T^{+} u=r_{S_{N}} T^{+} F_{1}-\left(r_{S_{N}} T^{+} \mathcal{R}_{+} u-r_{S_{N}} T^{+} \mathcal{R}_{-} v-r_{S_{N}} W^{\prime} \psi+r_{S_{N}} \mathscr{L}^{+} \varphi\right)$, $r_{S_{N}} T^{+} u=r_{S_{N}} T^{+} F_{1}-F_{4}$,

which coincides with the fourth equation in the MLBDIEs system (3.26)-(3.29),

$$
r_{S_{N}} T^{+} \mathcal{R}_{+} u-r_{S_{N}} T^{+} \mathcal{R}_{-} v-r_{S_{N}} \mathcal{W}^{\prime} \psi+r_{S_{N}} \mathscr{L}^{+} \varphi=F_{4} \quad \text { on } S_{N} .
$$

Thus we have shown that the MLBDIEs (3.26)-(3.29) are equivalent to the coupled mixed BVPs (3.76)-(3.78) and (3.79)-(3.81) in the above described sense.

Now the surjectivity of the operator (3.54) easily follows from the fact that the mixed BVPs (3.76)-(3.78) and (3.79)-(3.81) are solvable due to the Lax-Milgram theorem in the spaces $H_{2}^{1,0}\left(\Omega^{+}, L\right)$ and $X\left(\Omega^{-}, L\right)$, respectively, for arbitrary data (see, e.g., [6])

$$
\begin{gathered}
G \in L_{2}\left(\Omega^{+}\right), \quad\left(1+|x|^{2}\right)^{1 / 2} Q \in L_{2}\left(\Omega^{-}\right), \\
g_{1}, q_{1} \in H^{\frac{1}{2}}\left(S_{D}\right), \quad g_{2}, q_{2} \in H^{-\frac{1}{2}}\left(S_{N}\right) .
\end{gathered}
$$

This completes the proof.

\section{Bibliography}

[1] O. Chkadua, S. Mikhailov, and D. Natroshvili, Analysis of direct boundary-domain integral equations for a mixed BVP with variable coefficient. Part I. Equivalence and invertibility, J. Integral Equations Appl. 21:4 (2009), 499-542. 
[2] O. Chkadua, S. Mikhailov, and D. Natroshvili, Analysis of direct boundary-domain integral equations for a mixed BVP with variable coefficient. Part II. Solution regularity and asymptotics, J. Integral Equations Appl. 22:1 (2010), 19-37.

[3] O. Chkadua, S. Mikhailov, and D. Natroshvili, About analysis of some localized boundary-domain integral equations for a variable-coefficient BVP, in: Advances in Boundary Integral Methods. Proceedings of the 6th UK Conference on Boundary Integral Methods (Edited by J. Trevelyan), 291-302. Durham University Publ., UK, 2007.

[4] O. Chkadua, S. Mikhailov, and D. Natroshvili, Analysis of some localized boundarydomain integral equations, J. Integral Equations Appl. 21:3 (2009), 407-447.

[5] O. Chkadua, S. Mikhailov, and D. Natroshvili, Analysis of some boundary-domain integral equations for variable-coefficient problems with cracks, in: Advances in Boundary Integral Methods. Proceedings of the 7th UK Conference on Boundary Integral Methods, 37-51. Nottingham University Publ., UK, 2009.

[6] R. Dautray and J. L. Lions, Mathematical Analysis and Numerical Methods for Science and Technology. Vol. 4. Integral Equations and Numerical Methods, (Translated from the French) Springer-Verlag, Berlin, 1990.

[7] J.-L. Lions and E. Magenes, Non-homogeneous Boundary Value Problems and Applications, Vol. I (translated from the French), Die Grundlehren der mathematischen Wissenschaften Band 181. Springer-Verlag, New York-Heidelberg, 1972.

[8] S. Mikhailov, Localized boundary-domain integral formulation for problems with variable coefficients, Int. J. Engineering Analysis with Boundary Elements 26 (2002), 681-690.

[9] S. E. Mikhailov, Analysis of united boundary-domain integro-differential and integral equations for a mixed BVP with variable coefficient, Math. Methods Appl. Sci. 29:6 (2006), 715-739.

[10] C. Miranda, Partial Differential Equations of Elliptic Type, Second revised edition. (Translated from the Italian) Ergebnisse der Mathematik und ihrer Grenzgebiete, Band 2. Springer-Verlag, New York-Berlin, 1970.

[11] A. Pomp, The Boundary-Domain Integral Method for Elliptic Systems, With an application to shells. Lecture Notes in Mathematics, 1683. Springer-Verlag, Berlin, 1998.

[12] J. Sladek, V. Sladek, and S. N. Atluri, Local boundary integral equation (LBIE) method for solving problems of elasticity with nonhomogeneous material properties, Comput. Mech. 24:6 (2000), 456-462.

[13] A. E. Taigbenu, The Green Element Method, Kluwer Academic Publ,, Norwell, Massachusetts, 1999.

[14] V. S. Vladimirov, Generalized Functions in Mathematical Physics, (Russian) Generalized functions in mathematical physics, Nauka, Moscow, 1976. 
[15] T. Zhu, J.-D. Zhang, and S. N. Atluri, A local boundary integral equation (LBIE) method in computational mechanics, and a meshless discretization approach, Comput. Mech. 21:3 (1998), 223-235.

[16] T. Zhu, J.-D. Zhang, and S. N. Atluri, A meshless numerical method based on the local boundary integral equation (LBIE) to solve linear and non-linear boundary value problems, Eng. Anal. Bound. Elem. 23:5-6 (1999), 375-389.

Received July 3, 2009.

\section{Author information}

Otar Chkadua, A. Razmadze Mathematical Institute, 1, M. Aleksidze St., Tbilisi 0193, and Sokhumi State University, 9, Jikia st., Tbilisi 0186, Georgia.

E-mail: chkadua7@yahoo.com

Sergey E. Mikhailov, Department of Mathematics, Brunel University West London, Uxbridge, UB8 3PH, UK.

E-mail: sergey.mikhailov@brunel.ac.uk

David Natroshvili, Department of Mathematics, Georgian Technical University, 77, M. Kostava St., Tbilisi 0175, and I. Vekua Institute of Applied Mathematics of Tbilisi State University, 2, University St., Tbilisi 0186, Georgia.

E-mail: natrosh@hotmail.com 